\title{
Formulação de Elementos Finitos Estabilizados para Estruturas Flexíveis no Espaço 3D
}

A.J.B. SANTOS ${ }^{1}$, A.F.D. LOULA 2 , J.N.C. GUERREIRO ${ }^{3}$, Laboratório Nacional de Computação Científica, Av. Getúlio Vargas 333, 25651-075, Petrópolis, RJ, Brasil.

Resumo. Apresentamos um modelo computacional para estruturas flexíveis de geometria arbitrária no espaço tridimensional com a cinemática governada pela teoria de barras com flexão, cisalhamento, torção e efeito de membrana. Um método misto de Petrov-Galerkin é usado para construir uma aproximação estabilizada de elementos finitos. Estimativas de erro indicando taxas de convergência bem como resultados numéricos comprovando tais taxas são apresentados.

\section{Introdução}

Estruturas flexíveis, são largamente empregadas em muitos problemas de interesse prático na engenharia. Muitos desses problemas, como por exemplo, a análise de tensões de membros estruturais de pontes, edifícios altos e risers [6] para a extração de petróleo podem e são modelados com suficiente precisão como estruturas unidimensionais no espaço tridimensional. O interesse na modelagem desse tipo de estrutura tem seu crescimento continuado à medida em que se percebe potenciais aplicações em outras áreas do conhecimento como biomédica, microbiológia, biologia molecular e química [5].

O método dos elementos finitos, na sua forma clássica, baseada na formulação de Galerkin, é uma técnica bastante utilizada na obtenção de soluções aproximadas de problemas envolvendo estruturas flexíveis unidimensionais. Entretanto, as escolhas das ordens de interpolação dos campos envolvidos podem ser extremamente delicadas em estruturas delgadas modeladas por teorias com cortante bem como em estruturas curvas delgadas onde ocorrem acoplamentos entre esforços de membrana e de flexão. Observações experimentais mostram que deslocamentos calculados por escolhas inadequadas levam a valores menores que os reais.

Muitas investigações no sentido de sanar este problema já foram feitas explorando formulações de elementos finitos mistos na análise de estruturas unidimensionais planas (vigas e arcos planos) como podemos encontrar nos trabalhos [1], [7], [9] e [10]. Loula et al. estudaram esses problemas com a dependência de um parâmetro pequeno de esbeltez, e introduziram uma formulação mista de Petrov-Galerkin para a construção de aproximações discretas do problema da viga [8] e do arco

\footnotetext{
${ }^{1}$ boness@Incc.br. Agradeço à FAPERJ pelo suporte financeiro durante o curso deste trabalho.

2aloc@lncc.br

3joao@lncc.br
} 
de Timoshenko [7]. A formulação variacional mista por eles adotada baseia-se no princípio de Hellinger-Reissner no qual as tensões generalizadas (variavel primal) e os deslocamentos generalizados (multiplicadores) são as variáveis independentes do problema. Essa formulação, além de trazer ganhos na ordem de convergência, possibilita escolhas de elementos finitos de igual ordem para a interpolação dos campos de deslocamentos e tensões. Tais escolhas são instáveis na formulação de Galerkin.

Para estruturas unidimensionais, Arunakirinathar e Reddy [2] estenderam para o espaço tridimensional os estudos feitos por [9] e [10] para o caso de estruturas planas. Estes autores apresentaram uma formulação mista em que os deslocamentos generalizados são as variáveis principais e as tensões cisalhantes generalizadas os multiplicadores. Entretanto, como suas variáveis independentes são unicamente as tensões cisalhantes, essa formulação perde precisão no cálculo dos esforços de flexão e torção, pois estes são obtidos por cálculos indiretos.

Nesse trabalho, usando resultados de geometria diferencial apresentados por Arunakirinathar e Reddy [2], estendemos a metodologia desenvolvida por Loula et al. [7], para modelar o caso geral de uma barra flexível com geometria e carregamento arbitrário no espaço tridimensional usando a formulação mista de PetrovGalerkin. Na Seção 2 desse artigo descrevemos aspectos referentes à geometria do modelo e apresentamos funções intrínsecas baseadas na geometria diferencial de curvas. Na Seção 3 o sistema de equações que o descreve é apresentado na sua forma adimensional explicitando a dependência de um parâmetro pequeno $\varepsilon^{2}$. Sua cinemática baseia-se na teoria de barras com flexão, torção, efeito de membrana e cisalhamento que impõe as hipóteses das seções transversais da estrutura serem restritas a permanecerem planas, mas não necessariamente normais ao eixo de centróides, depois da deformação. Isso nos possibilita trabalhar com dois campos cinemáticos, deslocamento e rotação, reduzindo assim a ordem das derivadas das equações. Na Seção 4 construímos a formulação variacional contínua do modelo, discutimos e apresentamos os resultados de existência e unicidade de solução. Na Seção 5 apresentamos as aproximações de Galerkin e de Petrov-Galerkin para o problema e desenvolvemos a análise da formulação de Petrov-Galerkin estabelecendo existência e unicidade da solução discreta bem como estabilidade e convergência uniformes (independente do parâmetro $\varepsilon^{2}$ ). Através desta formulação estabilizada recuperamos a estabilidade e convergência das aproximações de igual ordem para esforços e deslocamentos generalizados instáveis na forma de Galerkin. Na Seção 6 apresentamos uma aplicação numérica comprovando os resultados da análise numérica desenvolvida.

\section{Geometria}

A configuração do eixo de centróides da estrutura é representada por uma curva regular $\mathbf{r}:(0, L) \rightarrow \mathbb{R}^{3}$ parametrizada por um comprimento de $\operatorname{arco} s \in(0, L) \subset \mathbb{R}$. Para cada valor de $s$ temos associados três vetores unitários e ortogonais $\mathbf{t}(s)$ vetor tangente, $\mathbf{n}(s)$ vetor normal e $\mathbf{b}(s)$ vetor binormal (Figura 1). O triedro formado por esses vetores no ponto $s$ é chamado de Triedro de Frenet. Suas derivadas com relação a $s, \mathbf{t}^{\prime}(s)=\kappa(s) \mathbf{n}(s), \mathbf{b}^{\prime}(s)=-\mu(s) \mathbf{n}(s)$ e $\mathbf{n}^{\prime}(s)=-\kappa(s) \mathbf{t}(s)-\mu(s) \mathbf{b}(s)$ expressas na base $\{\mathbf{t}(s), \mathbf{n}(s), \mathbf{b}(s)\}$ nos dão informações sobre o comportamento 
de $\mathbf{r}(s)$ na vizinhança de $s$ e são conhecidas como relações de Serret-Frenet. As funções geométricas $\kappa(s)>0$ e $\mu(s)>0$ são denominadas de curvatura e torção, respectivamente. Considerando $\mathbf{v}(s)=\left(v_{t}, v_{n}, v_{b}\right)$ uma função vetorial expressa na

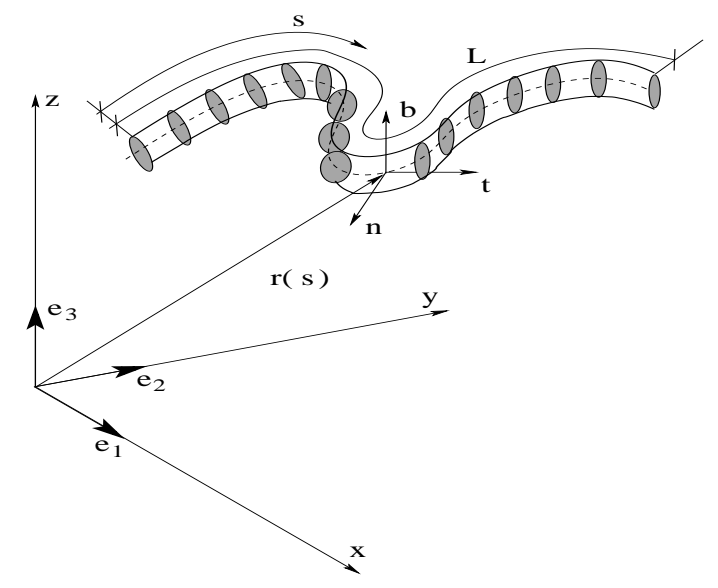

Figura 1: Modelo esquemático da estrutura flexível.

base $\{\mathbf{t}(s), \mathbf{n}(s), \mathbf{b}(s)\}$, utilizaremos a notação $\mathbf{v}^{\prime}(s)$ para nos referir a diferenciação tanto das componentes quanto dos vetores que compõem a base, isto é,

$$
\mathbf{v}^{\prime}(s)=\left(v_{t}^{\prime}-\kappa v_{n}\right) \mathbf{t}+\left(v_{n}^{\prime}+\kappa v_{t}-\mu v_{b}\right) \mathbf{n}+\left(v_{b}^{\prime}+\mu v_{n}\right) \mathbf{b} .
$$

\section{Modelo}

Este modelo simula o comportamento de uma estrutura flexível no espaço tridimensional e tem sua cinemática fundamentalmente governada pela teoria de barras com flexão, cisalhamento, torção e efeito de membrana.

A estrutura é considerada engastada em uma de suas extremidades e livre na outra, possui comprimento $L$, seção transversal $A$, momento polar de inércia $J$, momento de inércia $I_{n}$ e $I_{b}$ (em relação ao eixo $\mathbf{n}(s)$ e $\mathbf{b}(s)$, respectivamente), coeficiente de correção para compensar a distribuição não uniforme da tensão cisalhante $k_{n}$ e $k_{\theta}$ (em relação ao eixo $\mathbf{n}(s)$ e $\mathbf{b}(s)$, respectivamente), módulo de Young $E$, módulo cisalhante $G$ e está sujeita a cargas distribuídas por unidade de comprimento $F_{f}(s)=\left\{f_{t}, f_{n}, f_{b}\right\}^{t}$ e $F_{m}(s)=\left\{m_{t}, m_{n}, m_{b}\right\}^{t}$. Nós denotamos por $U(s)=\left\{u_{t}, u_{n}, u_{b}\right\}^{t}$ e $\phi=\left\{\phi_{t}, \phi_{n}, \phi_{b}\right\}^{t}$ os deslocamentos generalizados (deslocamento e rotações da linha de centróides) e $N(s)=\left\{N_{t}, N_{n}, N_{b}\right\}^{t}$ e $M(s)=\left\{M_{t}, M_{n}, M_{b}\right\}^{t}$ os esforços generalizados.

Para melhor explicitar as relações existentes entre o comprimento e a seção da 
estrutura faremos as seguintes adimensionalizações

$$
\begin{aligned}
& u_{1}=\frac{u_{t}}{L}, \quad u_{2}=\frac{u_{n}}{L}, \quad u_{3}=\frac{u_{b}}{L}, \quad \theta_{1}=\phi_{t}, \quad \theta_{2}=\phi_{n}, \quad \theta_{3}=\phi_{b}, \\
& \sigma_{1}=\frac{N_{t} L^{2}}{E I_{n}}, \quad \sigma_{2}=\frac{N_{n} L^{2}}{E I_{n}}, \quad \sigma_{3}=\frac{N_{b} L^{2}}{E I_{n}}, \sigma_{4}=\frac{M_{t} L}{E I_{n}}, \quad \sigma_{5}=\frac{M_{n} L}{E I_{n}}, \quad \sigma_{6}=\frac{M_{b} L}{E I_{n}}, \\
& f_{1}=\frac{f_{t} L^{3}}{E I_{n}}, \quad f_{2}=\frac{f_{n} L^{3}}{E I_{n}}, \quad f_{3}=\frac{f_{b} L^{3}}{E I_{n}}, \quad f_{4}=\frac{m_{t} L^{2}}{E I_{n}}, \quad f_{5}=\frac{m_{n} L^{2}}{E I_{n}}, \quad f_{6}=\frac{m_{b} L^{2}}{E I_{n}}
\end{aligned}
$$

e introduzimos os parâmetros

$$
\varepsilon^{2}=\frac{I_{n}}{L^{2} A} ; \quad \chi^{2}=\frac{E}{G k_{n}} ; \quad \rho^{2}=\frac{E}{G k_{b}} ; \quad \gamma^{2}=\frac{E I_{n}}{G J} ; \iota^{2}=\frac{I_{n}}{I_{b}} .
$$

A medida que a estrutura torna-se mais esbelta, o parâmetro $\varepsilon^{2}$ adquire valores cada vez menor $\left(\varepsilon^{2} \ll 1\right)$ enquanto que os parâmetros $\chi^{2}, \rho^{2}, \gamma^{2}$ e $\iota^{2}$ aproximam-se dada vez mais da ordem de um. Essas parametrizações conduzem ao seguinte problema adimensional:

Problema A: Dado $\mathbf{f}_{u}(s)=\left\{f_{1}, f_{2}, f_{3}\right\}^{t}$ e $\mathbf{f}_{\theta}(s)=\left\{f_{4}, f_{5}, f_{6}\right\}^{t}$, encontrar $\mathbf{u}(s)=$ $\left\{u_{1}, u_{2}, u_{3}\right\}^{t}, \boldsymbol{\theta}(s)=\left\{\theta_{1}, \theta_{2}, \theta_{3}\right\}^{t}, \boldsymbol{\sigma}_{u}(s)=\left\{\sigma_{1}, \sigma_{2}, \sigma_{3}\right\}^{t}$ e $\boldsymbol{\sigma}_{\theta}(s)=\left\{\sigma_{4}, \sigma_{5}, \sigma_{6}\right\}^{t}$, com $s \in(0,1)$ satisfazendo

Equações de equilíbrio

$$
\begin{aligned}
-\boldsymbol{\sigma}_{\theta}^{\prime}(s)+\boldsymbol{\sigma}_{u}(s) \wedge \mathbf{t}(s) & =\mathbf{f}_{\theta}(s), \\
-\boldsymbol{\sigma}_{u}^{\prime}(s) & =\mathbf{f}_{u}(s) .
\end{aligned}
$$

Equações constitutivas

$$
\begin{aligned}
\mathbf{E} \boldsymbol{\sigma}_{\theta}(s) & =\boldsymbol{\theta}^{\prime}(s), \\
\overline{\mathbf{D}} \boldsymbol{\sigma}_{u}(s) & =\mathbf{u}^{\prime}(s)-\boldsymbol{\theta}(s) \wedge \mathbf{t}(s) .
\end{aligned}
$$

\section{Condições de contorno}

$$
\begin{aligned}
& \mathbf{u}(0)=0, \\
& \boldsymbol{\theta}(0)=0,
\end{aligned}
$$

onde

$$
\begin{aligned}
& \mathbf{E}=\operatorname{diag}\left(\gamma^{2}, 1, \iota^{2}\right), \\
& \overline{\mathbf{D}}=\varepsilon^{2} \mathbf{D}, \\
& \mathbf{D}=\operatorname{diag}\left(1, \chi^{2} \rho^{2}\right)
\end{aligned}
$$

\section{Formulação Variacional}

Seja $L_{2}(0,1)$ o espaço de funções de quadrado integrável no sentido de Lebesgue e $H(0,1)$ o espaço de Hilbert

$$
H(0,1)=\left\{v ; v \in L_{2}(0,1), v^{\prime} \in L_{2}(0,1), v(0)=0\right\} .
$$


Com essas definições, identificamos os espaços de Hilbert $Q=\left(L_{2}(0,1)\right)^{3} \mathrm{e}$ $S=(H(0,1))^{3}$ com produto interno e norma dados, respectivamente, por

$$
\begin{aligned}
(\boldsymbol{\sigma}, \boldsymbol{\tau}) & =\int_{0}^{1} \boldsymbol{\sigma} \cdot \boldsymbol{\tau} d s ; \quad\|\boldsymbol{\sigma}\|=(\boldsymbol{\sigma}, \boldsymbol{\sigma})^{\frac{1}{2}} \quad \forall \boldsymbol{\sigma}, \boldsymbol{\tau} \in Q \\
(\mathbf{u}, \mathbf{v})_{1} & =\int_{0}^{1} \mathbf{u}^{\prime} \cdot \mathbf{v}^{\prime} d s ; \quad|\mathbf{v}|_{1}=(\mathbf{v}, \mathbf{v})_{1}^{\frac{1}{2}} \quad \forall \mathbf{u}, \mathbf{v} \in S
\end{aligned}
$$

onde é possível provar que a seminorma $|\cdot|_{1}$ é uma norma em $S$, e $W=Q \times Q$ e $V=S \times S$ com norma e produto interno dados, respectivamente, por

$$
\begin{aligned}
(\boldsymbol{\sigma}, \boldsymbol{\tau})=\left(\boldsymbol{\sigma}_{u}, \boldsymbol{\tau}_{u}\right)+\left(\boldsymbol{\sigma}_{\theta}, \boldsymbol{\tau}_{\theta}\right) ; & \|\boldsymbol{\tau}\|_{W}^{2}=\left\|\boldsymbol{\tau}_{u}\right\|^{2}+\left\|\boldsymbol{\tau}_{\theta}\right\|^{2}, \\
(\mathbf{z}, \mathbf{w})_{1}=\left(\mathbf{u}^{\prime}, \mathbf{v}^{\prime}\right)+\left(\boldsymbol{\theta}^{\prime}, \boldsymbol{\psi}^{\prime}\right) ; & \|\mathbf{w}\|_{V}^{2}=|\mathbf{v}|_{1}^{2}+|\boldsymbol{\psi}|_{1}^{2},
\end{aligned}
$$

onde

$$
\begin{array}{ll}
\mathbf{z}=\{\mathbf{u}, \boldsymbol{\theta}\}^{t} \in V, & \mathbf{w}=\{\mathbf{v}, \boldsymbol{\psi}\}^{t} \in V, \\
\boldsymbol{\sigma}=\left\{\boldsymbol{\sigma}_{u}, \boldsymbol{\sigma}_{\theta}\right\}^{t} \in W, & \boldsymbol{\tau}=\left\{\boldsymbol{\tau}_{u}, \boldsymbol{\tau}_{\theta}\right\}^{t} \in W .
\end{array}
$$

Considerando $\boldsymbol{\sigma} \in Q$ e $\mathbf{E}: W \rightarrow W$ um operador, definimos sua norma por

$$
\|\mathbf{E}\|=\max _{\boldsymbol{\sigma} \neq 0} \frac{\|\mathbf{E} \boldsymbol{\sigma}\|}{\|\boldsymbol{\sigma}\|} .
$$

Com isso, podemos apresentar o Problema A equivalentemente na forma variacional abstrata através do seguinte problema

Problema P: Dado $\left\{\mathbf{f}_{u}, \mathbf{f}_{\theta}\right\}^{t} \in V^{\prime}$, encontrar $\{\boldsymbol{\sigma}, \mathbf{z}\}^{t} \in W \times V$ tais que

$$
\begin{aligned}
a(\boldsymbol{\sigma}, \boldsymbol{\tau})+b(\mathbf{z}, \boldsymbol{\tau}) & =0 & & \forall \boldsymbol{\tau} \in W, \\
b(\boldsymbol{\sigma}, \mathbf{w}) & =f(\mathbf{w}) & & \forall \mathbf{w} \in V,
\end{aligned}
$$

onde $V^{\prime}$ é o espaço dual de $V$,

$$
\begin{aligned}
a(\boldsymbol{\sigma}, \boldsymbol{\tau}) & =-\left(\boldsymbol{E} \boldsymbol{\sigma}_{\theta}, \boldsymbol{\tau}_{\theta}\right)-\varepsilon^{2}\left(\boldsymbol{D} \boldsymbol{\sigma}_{u}, \boldsymbol{\tau}_{u}\right), \\
b(\boldsymbol{\tau}, \mathbf{z}) & =\left(\boldsymbol{\tau}_{\theta}, \boldsymbol{\theta}^{\prime}\right)+\left(\boldsymbol{\tau}_{u}, \mathbf{u}^{\prime}-\boldsymbol{\theta} \wedge \mathbf{t}\right) \\
f(\mathbf{z}) & =\left(\mathbf{f}_{u}, \mathbf{u}\right)+\left(\mathbf{f}_{\theta}, \boldsymbol{\theta}\right) .
\end{aligned}
$$

\subsection{Existência e unicidade de solução}

Sem perda de generalidade, consideraremos na análise $\varepsilon^{2} \ll 1$. O Problema $\mathbf{P}$ ajusta-se à classe de formulações mistas abstratas estudadas por Brezzi, Babŭska e outros. O teorema de Brezzi [3] afirma que esse problema tem solução única para qualquer valor de $\varepsilon$, inclusive o zero, se as propriedades de continuidade das formas $a(\cdot, \cdot)$ e $b(\cdot, \cdot)$, K-elipticidade de $a(\cdot, \cdot)$ e a condição de compatibilidade entre os espaços (LBB) forem garantidas.

Continuidade: As formas bilineares $a: W \times W \rightarrow \mathbb{R}$ e $b: W \times V \rightarrow \mathbb{R}$ são contínuas. Existem constantes positivas $\alpha_{1} \in \mathbb{R}$ e $\beta_{1} \in \mathbb{R}$ independente de $\varepsilon$ tais que

$$
\begin{array}{rll}
a(\boldsymbol{\sigma}, \boldsymbol{\tau}) & \leqslant \alpha_{1}\|\boldsymbol{\sigma}\|_{W}\|\boldsymbol{\tau}\|_{W} & \forall \boldsymbol{\sigma}, \boldsymbol{\tau} \in W, \\
b(\boldsymbol{\tau}, \mathbf{z}) \leqslant \beta_{1}\|\boldsymbol{\tau}\|_{W}\|\mathbf{z}\|_{V} & \forall \boldsymbol{\tau} \in W, \mathbf{z} \in V .
\end{array}
$$


K-elipticidade de $a(\cdot, \cdot)$ : Existe uma constante positiva $\alpha_{2} \in \mathbb{R}$ independente de $\varepsilon$ tal que

$$
a(\boldsymbol{\tau}, \boldsymbol{\tau}) \geqslant \alpha_{2}\|\boldsymbol{\tau}\|_{W}^{2} \quad \forall \boldsymbol{\tau} \in K
$$

onde

$$
K=\{\boldsymbol{\tau} \in W ; b(\boldsymbol{\tau}, \mathbf{z})=0 \quad \forall \mathbf{z} \in V\} .
$$

Condição LBB: Existe uma constante positiva $\beta_{2} \in \mathbb{R}$ independente de $\varepsilon$ tal que

$$
\sup _{\boldsymbol{\tau} \in W} \frac{b(\boldsymbol{\tau}, \mathbf{z})}{\|\boldsymbol{\tau}\|_{W}} \geqslant \beta_{2}\|\mathbf{z}\|_{V} \quad \forall \mathbf{z} \in V .
$$

Para o Problema $\mathbf{P}$ encontramos $\alpha_{1}=2\|\mathbf{E}\|, \alpha_{2}=\frac{\mathcal{M}_{E}}{2}\left(1-\frac{1}{\sqrt{2}}\right)^{2}, \beta_{1}=$ $2\left(1+\frac{1}{\sqrt{2}}\right)$ e $\beta_{2}=\frac{\sqrt{6}}{6}$, onde $\mathcal{M}_{E}=\min \left\{\gamma^{2}, 1, \iota^{2}\right\}$.

\section{Aproximações por Elementos Finitos}

Nessa seção, construímos e analisamos uma nova aproximação por elementos finitos do Problema P. Seja $I_{e}$ a partição de elementos finitos, $h_{e}$ o tamanho de $I_{e}$ e $n_{e l}$ o número total de elementos, denotamos por $h=\max \left\{h_{e}\right\}, 1 \leqslant e \leqslant n_{e l}$ o parâmetro de malha. Definindo

$$
Q_{h}=\left\{\boldsymbol{\tau}_{h} \in\left(C^{-1}(0,1)\right)^{3} ; \boldsymbol{\tau}_{h}\left(I_{e}\right) \in P_{l}\left(I_{e}\right)\right\}
$$

o espaço das funções descontínuas cuja restrição a $I_{e}$ são polinômios de grau $l$ e $S_{h}=Q_{h} \cap C^{0} \cap H(0,1)$ o espaço das funções contínuas cuja restrição ao elemento são polinômios de grau $l$, identificamos por $V_{h}=Q_{h} \times Q_{h} \subset V$ e $W_{h}=S_{h} \times S_{h} \subset W$ os espaços de aproximações para $W$ e $V$, respectivamente. Isso quer dizer que $\boldsymbol{\tau}_{h}$ e $\mathbf{z}_{h}$ são aproximações de igual ordem para $\boldsymbol{\tau}$ e $\mathbf{z}$, respectivamente, diferindo por $\boldsymbol{\tau}_{h}$ ser descontínuo e $\mathbf{z}_{h}$ ser contínuo no contorno dos elementos.

Com isso, a aproximação de Galerkin do Problema $\mathbf{P}$ é dada por

Problema $\mathbf{P}_{h}$ : Encontrar $\left\{\boldsymbol{\sigma}_{h}, \mathbf{z}_{h}\right\}^{t} \in W_{h} \times V_{h}$ tais que

$$
\begin{array}{rlrl}
a\left(\boldsymbol{\sigma}_{h}, \boldsymbol{\tau}_{h}\right)+b\left(\mathbf{z}_{h}, \boldsymbol{\tau}_{h}\right) & =0 & \forall \boldsymbol{\tau}_{h} \in W_{h}, \\
b\left(\boldsymbol{\sigma}_{h}, \mathbf{w}_{h}\right) & =f\left(\mathbf{w}_{h}\right) \quad \forall \mathbf{w}_{h} \in V_{h},
\end{array}
$$

onde

$$
\begin{aligned}
a\left(\boldsymbol{\sigma}_{h}, \boldsymbol{\tau}_{h}\right) & =-\left(\boldsymbol{E} \boldsymbol{\sigma}_{\theta h}, \boldsymbol{\tau}_{\theta h}\right)-\varepsilon^{2}\left(\boldsymbol{D} \boldsymbol{\sigma}_{u h}, \boldsymbol{\tau}_{u h}\right) \\
b\left(\boldsymbol{\tau}_{h}, \mathbf{z}_{h}\right) & =\left(\boldsymbol{\sigma}_{\theta h}, \boldsymbol{\theta}_{h}^{\prime}\right)+\left(\boldsymbol{\sigma}_{u h}, \mathbf{u}_{h}^{\prime}-\boldsymbol{\theta}_{h} \wedge \mathbf{t}\right) \\
f\left(\mathbf{z}_{h}\right) & =\left(\mathbf{f}_{u h}, \mathbf{u}_{h}\right)+\left(\mathbf{f}_{\theta h}, \boldsymbol{\theta}_{h}\right)
\end{aligned}
$$

A estabilidade dessa formulação está fundamentalmente atrelada à dimensão do parâmetro $\varepsilon$. Para valores grandes de $\varepsilon$, a formulação consegue aproximar a solução do problema sem maiores dificuldades, entretanto à medida em que esses valores diminuem complicações numéricas, como diminuição da precisão da solução e baixas taxas de convergência, começam a surgir até que no limite em que $\varepsilon \rightarrow 0$ 
há o trancamento (locking) onde a formulação perde completamente a capacidade de representar a solução. Isso se explica devido ao fato de não ser possível satisfazer à propriedade de K-elipticidade do operador $a(\cdot, \cdot)$ independente do parâmetro $\varepsilon$.

Uma forma de resolver este problema é aumentar a ordem dos polinômios de interpolação. Entretanto, isso acarreta taxas de convergências subótimas [8]. Uma outra forma, a qual seguiremos, é aproximar o Problema $\mathbf{P}$ por uma formulação de Petrov-Galerkin dada por

Problema PG: Encontrar $\left\{\boldsymbol{\sigma}_{h}, \mathbf{z}_{h}\right\}^{t} \in W_{h} \times V_{h}$ tais que

$$
\begin{aligned}
a_{h}\left(\boldsymbol{\sigma}_{h}, \boldsymbol{\tau}_{h}\right)+b\left(\mathbf{z}_{h}, \boldsymbol{\tau}_{h}\right) & =g_{h}\left(\boldsymbol{\tau}_{h}\right) \quad \forall \boldsymbol{\tau}_{h} \in W_{h}, \\
b\left(\boldsymbol{\sigma}_{h}, \mathbf{w}_{h}\right) & =f\left(\mathbf{w}_{h}\right) \quad \forall \mathbf{w}_{h} \in V_{h},
\end{aligned}
$$

onde

$$
\begin{aligned}
a_{h}\left(\boldsymbol{\sigma}_{h}, \boldsymbol{\tau}_{h}\right) & =a\left(\boldsymbol{\sigma}_{h}, \boldsymbol{\tau}_{h}\right)+a_{\delta}\left(\boldsymbol{\sigma}_{h}, \boldsymbol{\tau}_{h}\right)_{h} \\
a_{\delta}\left(\boldsymbol{\sigma}_{h}, \boldsymbol{\tau}_{h}\right)_{h} & =\sum_{e=1}^{n_{e l}} a_{e}\left(\boldsymbol{\sigma}_{e}, \boldsymbol{\tau}_{e}\right) \\
a_{e}\left(\boldsymbol{\sigma}_{e}, \boldsymbol{\tau}_{e}\right) & =-\delta_{1} h_{e}^{2}\left(\boldsymbol{\sigma}_{\theta e}^{\prime}-\boldsymbol{\sigma}_{u e} \wedge \mathbf{t}, \boldsymbol{\tau}_{\theta e}^{\prime}-\boldsymbol{\tau}_{u e} \wedge \mathbf{t}\right)-\delta_{2} h_{e}^{2}\left(\boldsymbol{\sigma}_{u e}^{\prime}, \boldsymbol{\tau}_{u e}^{\prime}\right) \\
b\left(\boldsymbol{\tau}_{h}, \mathbf{z}_{h}\right) & =\left(\boldsymbol{\sigma}_{\theta h}, \boldsymbol{\theta}_{h}^{\prime}\right)+\left(\boldsymbol{\sigma}_{u h}, \mathbf{u}_{h}^{\prime}-\boldsymbol{\theta}_{h} \wedge \mathbf{t}\right) \\
f\left(\mathbf{z}_{h}\right) & =\left(\mathbf{f}_{u h}, \mathbf{u}_{h}\right)+\left(\mathbf{f}_{\theta h}, \boldsymbol{\theta}_{h}\right) \\
g_{h}\left(\boldsymbol{\tau}_{h}\right) & =\sum_{e=1}^{n_{e l}} g_{e}\left(\boldsymbol{\tau}_{e}\right) \\
g_{e}\left(\boldsymbol{\tau}_{e}\right) & =\left(\mathbf{f}_{\theta e}, \boldsymbol{\tau}_{\theta e}^{\prime}-\boldsymbol{\tau}_{u e} \wedge \mathbf{t}\right)+\left(\mathbf{f}_{u e}, \boldsymbol{\tau}_{u e}^{\prime}\right)
\end{aligned}
$$

$\boldsymbol{\tau}_{e}$ representa a restrição de $\boldsymbol{\tau}_{h}$ a $I_{e}, \delta_{1} \geqslant 0$ e $\delta_{2} \geqslant 0$ constantes.

Como podemos perceber pela equação $(5.1)$, a forma $a_{h}(\cdot, \cdot)$ possui, além da forma discreta de $a(\boldsymbol{\sigma}, \boldsymbol{\tau})$, um termo adicional que desempenha um papel estabilizador. Esse termo provém de uma adição consistente de formas residuais de mínimos quadrados das equações de equilíbrio do problema.

A existência e unicidade de solução do Problema PG independente de $\varepsilon$ é garantida pela forma discreta do teorema de Brezzi [3]. Como $V_{h} \subset V$ e $W_{h} \subset$ $W$ a propriedade de continuidade da forma $b(\cdot, \cdot)$ é herdada da forma contínua. Entretanto, em geral $K_{h} \not \subset K$ e com isso todas as outras condições do caso contínuo devem ser verificadas no caso discreto.

Lema 5.1. Para todo $\boldsymbol{\tau}_{\theta h} \in Q_{h}$ existe uma constante positiva $C \in \mathbb{R}$ independente de $\varepsilon$ e h tal que

$$
\left\|\boldsymbol{\tau}_{\theta e}^{\prime}\right\|^{2} \leqslant \frac{C}{h_{e}^{2}}\left\|\boldsymbol{\tau}_{\theta e}\right\|^{2}
$$

onde $\boldsymbol{\tau}_{\theta e}$ é a restrição de $\boldsymbol{\tau}_{\theta h}$ a $I_{e}$.

Demonstração. Usando a definição

$$
\left\|\boldsymbol{\tau}_{\theta e}^{\prime}\right\|^{2}=\left\|\tau_{4 e}^{\prime}\right\|^{2}+\left\|\tau_{5 e}^{\prime}\right\|^{2}+\left\|\tau_{6 e}^{\prime}\right\|^{2}
$$


e a estimativa inversa

$$
\left\|\zeta_{i e}^{\prime}\right\|^{2} \leqslant \frac{C}{h_{e}^{2}}\left\|\zeta_{i e}\right\|^{2} \quad i=4,5,6
$$

apresentada em [4], para as funções $\tau_{i e}$ obtemos (5.8).

Lema 5.2 (Continuidade de $a_{h}(\cdot, \cdot)$ ). Existe uma constante positiva $\alpha_{3} \in \mathbb{R}$ independente de $\varepsilon$ e $h$ tal que

$$
a_{h}\left(\boldsymbol{\sigma}_{h}, \boldsymbol{\tau}_{h}\right) \leqslant \alpha_{3}\left\|\boldsymbol{\sigma}_{h}\right\|_{W}\left\|\boldsymbol{\tau}_{h}\right\|_{W} \quad \forall \boldsymbol{\sigma}_{h}, \boldsymbol{\tau}_{h} \in W_{h} .
$$

Demonstração. Pela definição (5.1) temos

$$
\left|a_{h}\left(\boldsymbol{\sigma}_{h}, \boldsymbol{\tau}_{h}\right)\right| \leqslant\left|a\left(\boldsymbol{\sigma}_{h}, \boldsymbol{\tau}_{h}\right)\right|+\sum_{e=1}^{n_{e l}}\left|a_{e}\left(\boldsymbol{\sigma}_{e}, \boldsymbol{\tau}_{e}\right)\right| .
$$

Analisando separadamente os dois termos do lado direito observamos que o primeiro tem sua continuidade garantida diretamente pela forma contínua (4.3)

$$
\left|a\left(\boldsymbol{\sigma}_{h}, \boldsymbol{\tau}_{h}\right)\right| \leqslant 2\|\mathbf{E}\|\left\|\boldsymbol{\sigma}_{h}\right\|_{W}\left\|\boldsymbol{\tau}_{h}\right\|_{W} \quad \forall \boldsymbol{\sigma}_{h}, \boldsymbol{\tau}_{h} \in W_{h} .
$$

Para o segundo termo temos por (5.3)

$$
\left|a_{e}\left(\boldsymbol{\sigma}_{e}, \boldsymbol{\tau}_{e}\right)\right| \leqslant \delta_{1} h_{e}^{2}\left(\left\|\boldsymbol{\sigma}_{\theta e}^{\prime}\right\|+\left\|\boldsymbol{\sigma}_{u e} \wedge \mathbf{t}\right\|\right)\left(\left\|\boldsymbol{\tau}_{\theta e}^{\prime}\right\|+\left\|\boldsymbol{\tau}_{u e} \wedge \mathbf{t}\right\|\right)+\delta_{2} h_{e}^{2}\left\|\boldsymbol{\sigma}_{u e}^{\prime}\right\|\left\|\boldsymbol{\tau}_{u e}^{\prime}\right\|
$$

e fazendo uso da desigualdade (5.8) obtemos

$$
\left|a_{e}\left(\boldsymbol{\sigma}_{e}, \boldsymbol{\tau}_{e}\right)\right| \leqslant 8 c_{1}\left(h_{e}+C\right)^{2}\left\|\boldsymbol{\sigma}_{e}\right\|_{W}\left\|\boldsymbol{\tau}_{e}\right\|_{W},
$$

onde $c_{1}=\max \left\{\delta_{1}, \delta_{2}\right\}$. Aplicando (5.12) e (5.11) em (5.10) encontramos (5.9) com $\alpha_{3}=\max \left\{2\|\mathbf{E}\|, 8 c_{1}\left(h_{e}+C\right)^{2}\right\}$.

Lema 5.3 (A LBB discreta). Existe uma constante positiva $\beta_{3} \in \mathbb{R}$ independente de $\varepsilon$ e $h$ tal que

$$
\sup _{\boldsymbol{\tau}_{h} \in W_{h}} \frac{b\left(\boldsymbol{\tau}_{h}, \mathbf{z}_{h}\right)}{\left\|\boldsymbol{\tau}_{h}\right\|_{W}} \geqslant \beta_{3}\left\|\mathbf{z}_{h}\right\|_{V} \quad \forall \mathbf{z}_{h} \in V_{h} .
$$

Demonstração. Todo $\widetilde{\boldsymbol{\tau}}_{h} \in W_{h}$ satisfaz à desigualdade

$$
\sup _{\boldsymbol{\tau}_{h} \in W_{h}} \frac{b\left(\boldsymbol{\tau}_{h}, \mathbf{z}_{h}\right)}{\left\|\boldsymbol{\tau}_{h}\right\|_{W}} \geqslant \frac{b\left(\widetilde{\boldsymbol{\tau}}_{h}, \mathbf{z}_{h}\right)}{\left\|\widetilde{\boldsymbol{\tau}}_{h}\right\|_{W}} \quad \forall \mathbf{z}_{h} \in V_{h} .
$$

Por (5.4), temos que para todo $\widetilde{\boldsymbol{\tau}}_{h} \in W_{h}$

$$
b\left(\widetilde{\boldsymbol{\tau}}_{h}, \mathbf{z}_{h}\right)=\left(\widetilde{\boldsymbol{\tau}}_{\theta h}, \boldsymbol{\theta}_{h}^{\prime}\right)+\left(\widetilde{\boldsymbol{\tau}}_{u h}, \mathbf{u}_{h}^{\prime}-\boldsymbol{\theta}_{h} \wedge \mathbf{t}\right) \quad \forall \mathbf{z}_{h} \in V_{h} .
$$

Escolhendo

$$
\begin{aligned}
& \widetilde{\boldsymbol{\tau}}_{\theta h}=\boldsymbol{\theta}_{h}^{\prime}, \\
& \widetilde{\boldsymbol{\tau}}_{u h}=\mathbf{u}_{h}^{\prime}-\boldsymbol{\theta}_{h} \wedge \mathbf{t},
\end{aligned}
$$


obtemos

$$
b\left(\widetilde{\boldsymbol{\tau}}_{h}, \mathbf{z}_{h}\right) \geqslant \frac{1}{2}\left\|\mathbf{z}_{h}\right\|_{V}^{2}
$$

Por outro lado, usando (5.15), (5.16) e a desigualdade de Poincaré

$$
\left\|\boldsymbol{\theta}_{h}(s)\right\|^{2} \leqslant \frac{1}{2}\left|\boldsymbol{\theta}_{h}(s)\right|_{1}^{2}
$$

de (4.2) encontramos

$$
\left\|\widetilde{\boldsymbol{\tau}}_{h}\right\|_{W}^{2} \leqslant \frac{3}{2}\left\|\mathbf{z}_{h}\right\|^{2}
$$

Aplicando (5.17) e (5.18) em (5.14)

$$
\sup _{\boldsymbol{\tau}_{h} \in W_{h}} \frac{b\left(\boldsymbol{\tau}_{h}, \mathbf{z}_{h}\right)}{\left\|\boldsymbol{\tau}_{h}\right\|_{W}} \geqslant \frac{b\left(\widetilde{\boldsymbol{\tau}}_{h}, \mathbf{z}_{h}\right)}{\left\|\widetilde{\boldsymbol{\tau}}_{h}\right\|_{W}} \geqslant \frac{\sqrt{6}\left\|\mathbf{z}_{h}\right\|}{6} \quad \forall \mathbf{z}_{h} \in V_{h}
$$

demonstramos (5.13) $\operatorname{com} \beta_{3}=\frac{\sqrt{6}}{6}$.

Lema 5.4. Para todo $\tau_{u h} \in Q_{h}$ a seguinte desigualdade é sempre satisfeita

$$
h^{2} \sum_{e=1}^{n_{e l}}\left(\boldsymbol{\tau}_{u e}^{\prime}, \boldsymbol{\tau}_{u e}^{\prime}\right) \geqslant 2\left\|\boldsymbol{\tau}_{u h}^{*}\right\|^{2} .
$$

Demonstração. Toda função $\tau_{u h} \in Q_{h}$ pode ser decomposta unicamente em

$$
\boldsymbol{\tau}_{u h}=\boldsymbol{\tau}_{u h}^{*}+\overline{\boldsymbol{\tau}}_{u h}
$$

onde $\overline{\boldsymbol{\tau}}_{u h}$ são funções constantes por parte e $\boldsymbol{\tau}_{u h}^{*}$ funções de média nula. Definindo $\overline{\boldsymbol{\tau}}_{u e}$ a restrição de $\overline{\boldsymbol{\tau}}_{u h}$ a $I_{e}$ por

$$
\overline{\boldsymbol{\tau}}_{u e}=\frac{1}{h_{e}} \int_{0}^{h_{e}} \boldsymbol{\tau}_{u e}(s) d s,
$$

temos que $\boldsymbol{\tau}_{u e}^{*}$, a restrição de $\boldsymbol{\tau}_{u h}^{*}$ a $I_{e}$, satisfaz

$$
\int_{0}^{\boldsymbol{h}_{e}} \boldsymbol{\tau}_{u e}^{*}(s) d s=0 \quad e=1, \ldots, n_{e l .}
$$

Considerando $t, s \in\left[0, h_{e}\right]$, onde $t$ é um ponto onde $\boldsymbol{\tau}_{u e}^{*}(s)$ inverte o sinal, $\boldsymbol{\tau}_{u h}^{*}(t)=$ 0 , temos

$$
\left|\boldsymbol{\tau}_{u e}^{*}(s)\right| \leqslant \int_{t}^{s}\left|\boldsymbol{\tau}_{u e}^{* \prime}(\xi)\right| d \xi
$$

Como $\overline{\boldsymbol{\tau}}_{u e}$ a nível de elemento é constante, derivando (5.20) garantimos a identidade $\boldsymbol{\tau}_{u e}^{\prime}=\boldsymbol{\tau}_{u e}^{* \prime}$ a qual aplicando em (5.21) nos garante (5.19). 
Lema 5.5 (K-elipticidade de $\left.a_{h}(\cdot, \cdot)\right)$. Existe uma constante positiva $\alpha_{4} \in \mathbb{R}$ independente de $\varepsilon$ e $h$ tal que

$$
a_{h}\left(\boldsymbol{\tau}_{\boldsymbol{h}}, \boldsymbol{\tau}_{\boldsymbol{h}}\right) \geqslant \alpha_{4}\left\|\boldsymbol{\tau}_{\boldsymbol{h}}\right\|_{W}^{2} \quad \forall \boldsymbol{\tau}_{\boldsymbol{h}} \in K_{h},
$$

onde

$$
K_{h}=\left\{\boldsymbol{\tau}_{h} \in W_{h} ; b\left(\boldsymbol{\tau}_{h}, \mathbf{w}_{h}\right)=0 \quad \forall \mathbf{w}_{h} \in V_{h}\right\} .
$$

Demonstração. Pela definição $(5.1-5.3)$ temos que

$$
\begin{aligned}
\left|a_{h}\left(\boldsymbol{\tau}_{h}, \boldsymbol{\tau}_{h}\right)\right| \geqslant & \mathcal{M}_{E}\left\|\boldsymbol{\tau}_{\theta h}\right\|^{2}+\varepsilon^{2} \mathcal{M}_{D}\left\|\boldsymbol{\tau}_{u h}\right\|^{2}+ \\
& \sum_{e=1}^{n_{e l}}\left(\delta_{1} h_{e}^{2}\left\|\boldsymbol{\tau}_{\theta e}^{\prime}-\boldsymbol{\tau}_{u e} \wedge \mathbf{t}\right\|^{2}+\delta_{2} h_{e}^{2}\left\|\boldsymbol{\tau}_{u e}^{\prime}\right\|^{2}\right),
\end{aligned}
$$

onde $\mathcal{M}_{D}=\min \left\{1, \chi^{2}, \rho^{2}\right\}$. Desconsiderando o termo dependente do parâmetro $\varepsilon$, o termo positivo associado ao resíduo da equação de equilíbrio dos momentos e aplicando (5.19) obtemos

$$
\left|a_{h}\left(\boldsymbol{\tau}_{h}, \boldsymbol{\tau}_{h}\right)\right| \geqslant \mathcal{M}_{E}\left\|\boldsymbol{\tau}_{\theta h}\right\|^{2}+2 \delta_{2}\left\|\boldsymbol{\tau}_{u h}^{*}\right\|^{2} .
$$

Supondo válida a desigualdade

$$
\frac{1}{2}\left\|\overline{\boldsymbol{\tau}}_{u h}\right\|^{2} \leqslant\left\|\boldsymbol{\tau}_{u h}^{*}\right\|^{2}+\left\|\boldsymbol{\tau}_{\theta h}\right\|^{2}
$$

e aplicando-a em (5.24) obtemos (5.22) com

$$
\alpha_{4}=\min \left\{\frac{\mathcal{M}_{E}}{4}, \frac{\delta_{2}}{2}\right\} .
$$

Para completar a prova resta apenas provar a relação (5.25). Pela definição (5.23) temos que todo $\boldsymbol{\tau}_{h} \in K_{h}$ satisfaz

$$
\left(\boldsymbol{\tau}_{\theta h}, \boldsymbol{\psi}_{h}^{\prime}\right)+\left(\boldsymbol{\tau}_{u h}, \mathbf{v}_{h}^{\prime}-\boldsymbol{\psi}_{h} \wedge \mathbf{t}\right)=0 \quad \forall\left(\mathbf{v}_{h}, \boldsymbol{\psi}_{h}\right) \in V_{h} .
$$

Aplicando o módulo em ambos os lados e fazendo uso da desigualdade de Poincaré obtemos

$$
\left|\left(\boldsymbol{\tau}_{u h}, \mathbf{v}_{h}^{\prime}\right)\right| \leqslant \frac{1}{\sqrt{2}}\left\|\boldsymbol{\tau}_{u h}\right\|\left\|\boldsymbol{\psi}_{h}^{\prime}\right\|+\left\|\boldsymbol{\tau}_{\theta h}\right\|\left\|\boldsymbol{\psi}_{h}^{\prime}\right\| .
$$

Desde que cada componente de $\overline{\boldsymbol{\tau}}_{u h}$ seja constante por partes e cada componente de $\mathbf{v}_{h}$ e $\boldsymbol{\psi}_{h}$ sejam no mínimo lineares, podemos escolher

$$
\begin{aligned}
\mathbf{v}_{h}(s) & =\int_{0}^{s} \overline{\boldsymbol{\tau}}_{u h}(\xi) d \xi, \\
\boldsymbol{\psi}_{h}(s) & =\int_{0}^{s} \frac{1}{\sqrt{2}} \overline{\boldsymbol{\tau}}_{u h}(\xi) d \xi .
\end{aligned}
$$

Usando as escolhas acima e a identidade (5.20) na desigualdade (5.27) obtemos a relação (5.25) completando assim a prova da $K_{h}$-elipticidade de $a_{h}(\cdot, \cdot)$. 
Teorema 5.1. Para $\delta_{2}>0$ o Problema PG tem solução única $\left\{\boldsymbol{\sigma}_{h}, \mathbf{u}_{h}\right\}^{t} \in$ $W_{h} \times V_{h}$ e vale a seguinte estimativa

$$
\left\|\boldsymbol{\sigma}-\boldsymbol{\sigma}_{h}\right\|_{W}+\left\|\mathbf{z}-\mathbf{z}_{h}\right\|_{V} \leqslant c\left(\left\|\boldsymbol{\sigma}-\boldsymbol{\tau}_{h}\right\|_{W}+\left\|\mathbf{z}-\mathbf{w}_{h}\right\|_{V}\right) \forall \mathbf{w}_{h} \in V_{h}, \boldsymbol{\tau}_{h} \in W_{h},
$$

onde c é constante e independente de $\varepsilon$ e $h$. Para $\{\boldsymbol{\sigma}, \mathbf{u}\}^{t}$ solução do Problema $\mathbf{P}$, suficientemente regular resulta

$$
\left\|\boldsymbol{\sigma}-\boldsymbol{\sigma}_{h}\right\|_{W}+\left\|\mathbf{z}-\mathbf{z}_{h}\right\|_{V} \leqslant c h^{l+1}\|\boldsymbol{\sigma}\|_{l+1}+c h^{l}\|\mathbf{z}\|_{l+1} .
$$

A prova é conseqüência direta da continuidade de $a_{h}$ (Lema 1), da LBB discreta (Lema 3), da K-elipticidade de $a_{h}$ (Lema 5), do teorema de Brezzi [3] e de resultados de teorias de interpolações de elementos finitos [4]. A equação (5.29) mostra que os deslocamentos e as tensões generalizadas convergem, respectivamente, com taxas ótimas e quase ótimas.

\section{Experimento Numérico}

A fim de comprovar numericamente os resultados da análise, consideramos a geometria da linha de centróides da estrutura descrevendo uma hélice no espaço, definida pela curva $r(s)=\left(\frac{\sqrt{2}}{4 \pi} \cos (2 \pi s), \frac{\sqrt{2}}{4 \pi} \operatorname{sen}(2 \pi s), \frac{\sqrt{2} s}{2}\right)$, engastada em uma extremidade e livre na outra, com parâmetros geométricos e constitutivos $\kappa=$ $\mu=\sqrt{2} \pi, \gamma^{2}=\iota^{2}=\chi^{2}=\rho^{2}=1, \varepsilon^{2}=10^{-8}$ e sujeita a uma carga unitária $P=-\frac{1}{\sqrt{2}} \mathbf{t}-\frac{1}{\sqrt{2}} \mathbf{b}$ distribuída uniformemente.

Cada uma das Figuras 2 e 3 são compostas por dois gráficos que visam caracterizar a influência da adição de termos de mínimos quadrados à formulação do problema aproximado. Cada gráfico contém estudos de convergências confrontando, para o mesmo problema, a solução da formulação de Galerkin com a de Petrov-Galerkin com interpolações de mesma ordem para os campos de deslocamentos e tensões. Os elementos comparados são: (G 2) e (G 3) que representam, respectivamente, interpolações lineares e quadráticas para a formulação de Galerkin (obtida fazendo $\left.\delta_{1}=\delta_{2}=0\right)$ e (PG 2) e (PG 3) que representam, respectivamente, interpolações lineares e quadráticas para a formulação de Petrov-Galerkin (obtida fazendo $\delta_{1}=\delta_{2}=1$ ).

Nos gráficos da Figura 2 as variáveis estudadas foram o deslocamento na direção do eixo normal e a rotação em relação ao mesmo eixo. Como podemos constatar, o caso ( $\mathrm{G}$ 2) não apresenta convergência. $\mathrm{O}$ caso ( $\mathrm{G}$ 3) apresenta no final um princípio de convergência, mas com o valor do erro inaceitável para a ordem de interpolação e ainda suscetível a piora se diminuirmos ainda mais o valor de $\varepsilon^{2}$. Em contrapartida os casos (PG 2) e (PG 3) confirmaram os resultados obtido na análise com taxa ótima (linear e quadrática, respectivamente, para interpolações lineares e quadráticas na norma $W$ ).

Nos gráficos da Figura 3 estudamos os esforços onde as variáveis foram a tensão cisalhante na direção do eixo normal $\left(\sigma_{h 2}\right)$ e o momento em relação ao mesmo eixo $\left(\sigma_{h 5}\right)$. No caso (G 2$)$ e (G 3$)$ podemos observar que $\left(\sigma_{h 5}\right)$ apresenta os mesmos comportamentos do deslocamento, entretanto em $\left(\sigma_{h 2}\right)$ há o surgimento de oscilações 

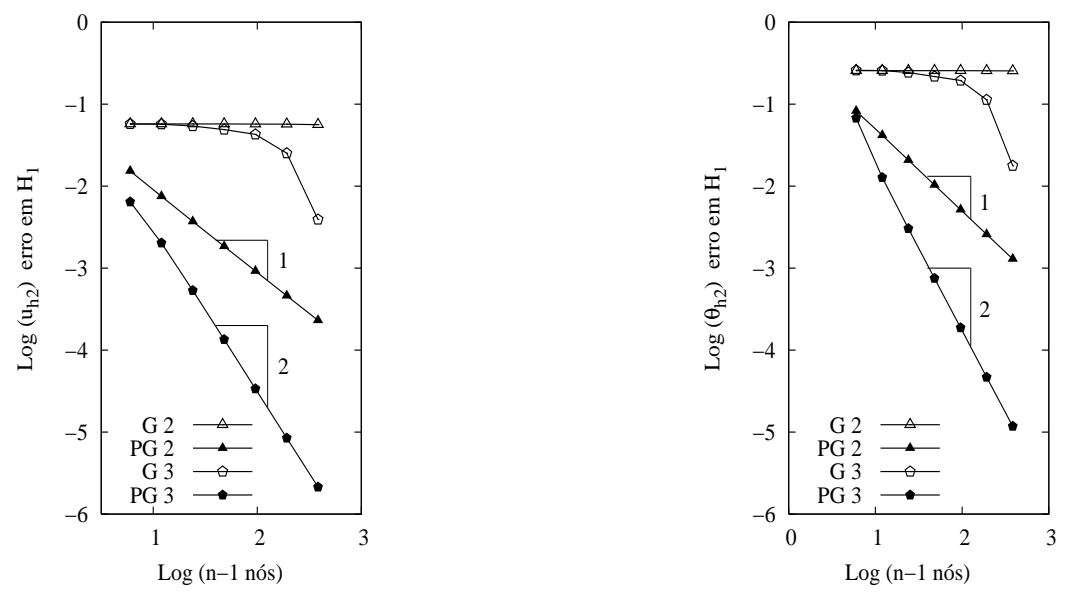

Figura 2: Estudos de convergências para $u_{h 2}$ e $\theta_{h 2}$.

fazendo com que (G 2) e (G 3) divirjam. Os casos (PG 2) e (PG 3) confirmaram os resultados obtido na análise com taxa subótimas (linear e quadrática, respectivamente, para interpolações lineares e quadráticas na norma $V$ ).

Abstract. We present a computational model for unidimensional flexible structures of arbitrary geometry in the three-dimensional space. The kinematics is given by bar theory and considers flexion, shear, torsion and membrane effects. A mixed Petrov-Galerkin method is used to construct a stable approximation of finite elements. Error estimates indicating convergence rates as well as numerical experiments that confirm these rates are presented.

\section{Referências}

[1] D.N. Arnold, Discretization by finite elements of a model parameter dependent problem, Numer. Math., 16 (1981), 405-421.

[2] K. Arunakirinathar and B.D. Reddy, Mixed finite element method for elastic rods of arbitrary geometry, Numer. Math., 16 (1993), 13-43.

[3] F. Brezzi, On the existence, uniqueness and approximation of saddle-point problems arising from lagrange mutipliers, RAIRO Ser. Rouge Anal. Numer., 8 (1974), 129-151.

[4] P.G. Ciarlet, "The Finite Elements of Elliptic Problem", North-Holland, Amsterdam, 1978.

[5] B. D. Coleman, D. Swigon, Theory of supercoiled elastic rings with self-contact and its appliations to dna plasmids, Journal of Elasticity, 60 (2000), 173-221.

[6] A. B. Custódio, "Modelo Analítico para Avaliação de Instabilidade nas Armaduras de Dutos Flexíveis", Tese, Engenharia Oceânica, UFRJ, Rio de Janeiro, Brasil, Out 2005. 

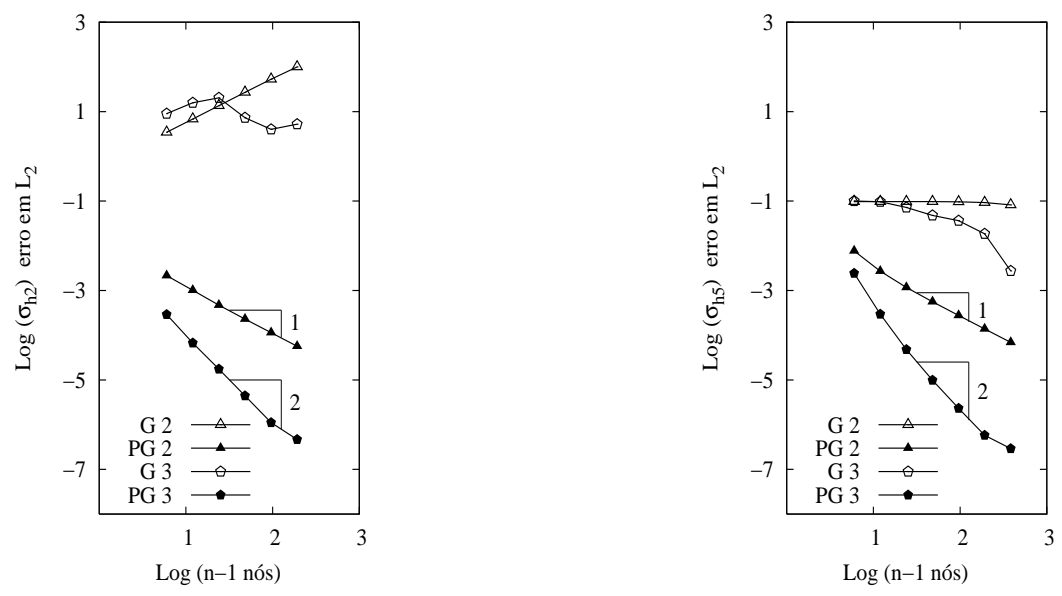

Figura 3: Estudos de convergências para $\sigma_{h 2}$ e $\sigma_{h 5}$.

[7] A.F.D. Loula, L.P. Franca, J.R. Hughes, I. Miranda, Stability, convergence and accuracy of a new finite element method for the circular arch problem, Comp. Meth. Appl. Mech. Engng., 63 (1987), 281-303.

[8] A.F.D. Loula, J.R. Hughes, L.P. Franca, J.R. Hughes, I. Miranda, Mixed petrov-galerkin methods for timoshenko beam problem, Comp. Meth. Appl. Mech. Engng., 63 (1987), 133-154.

[9] B.D. Reddy, Convergence of mixed finite element method approximation for the shallow arch problem, Numer. Math., 53 (1988), 687-699.

[10] B.D. Reddy, M.B. Volpi, Mixed finite element method for the arch problem, Comp. Meth. Appl. Mech. Engng., 97 (1992), 125-145. 
